\title{
Análise bibliométrica sobre o uso de biopolímeros pela indústria da construção civil
}

\author{
Bibliometric analysis on the use of biopolymers by the civil construction industry \\ Análisis bibliométrico sobre el uso de biopolímeros por la industria de la construcción civil
}

Recebido: 17/03/2021 | Revisado: 26/03/2021 | Aceito: 27/03/2021 | Publicado: 04/04/2021

\author{
Arilmara Abade Bandeira \\ ORCID: https://orcid.org/0000-0001-6393-6242 \\ Universidade Federal de Sergipe, Brasil \\ E-mail: arilmara2015@gmail.com \\ Wilsonita de Melo Ubirajara \\ ORCID: https://orcid.org/0000-0001-8643-6347 \\ Universidade Federal de Sergipe, Brasil \\ E-mail: will_ubirajara@yahoo.com.br \\ Francisco Sandro Rodrigues Holanda \\ ORCID: https://orcid.org/0000-0003-3575-8105 \\ Universidade Federal de Sergipe, Brasil \\ E-mail: fholanda@infonet.com.br \\ Carlos Eduardo Celestino de Andrade \\ ORCID: https://orcid.org/0000-0001-9336-1072 \\ Universidade Federal de Sergipe, Brasil \\ E-mail: eng2carlos.eduardo@gmail.com \\ Luiz Diego Vidal Santos \\ ORCID: https://orcid.org/0000-0001-8659-8557 \\ Universidade Federal de Sergipe, Brasil \\ E-mail: vidal.center@academico.ufs.br
}

\begin{abstract}
Resumo
As exigências oriundas do contexto socioambiental servem de estímulo para a inovação de produtos e processos pela indústria da construção civil. Uma possibilidade em crescimento é a utilização de materiais a base de biopolímeros, que são obtidos por rotas não convencionais de tranformação e não derivados do petróleo. Neste cenário, o objetivo do estudo foi identificar a produção científica relativa aos biopolímeros empregados na indústria da construção através de uma análise bibliométrica para o período de 2010 a 2020. O repositório de dados utilizados foi o Scopus Elsevier, aplicando os filtros em atenção aos critérios de inclusão e exclusão. Os resultados apontam que as publicações estão distribuídas em periódicos das áreas de Engenharia e também naqueles relacionados à Sustentabilidade Ambiental e que os autores mais citados, Ilhan Chang, Gye-Chun Cho e Jooyoung Im, foram também os mais produtivos e influentes sobre o tema. A maior contribuição científica sobre o uso de biopolímeros na construção civil foi dada pelos Estados Unidos, seguido por Índia e Coréia do Sul. Pode-se observar um aumento significativo do número de publicações a partir de 2019, o que demonstra a importância que a comunidade científica passou a dar ao tema a partir de então e também a distribuição das publicações entre os diversos países e instituições de pesquisa, demonstrando o interesse por essa área nos diversos continentes.
\end{abstract}

Palavras-chave: Inovação; Matérias primas; Derivados do petróleo; Socioambiental.

\begin{abstract}
The demands arising from the socio-environmental context serve as a stimulus for the innovation of products and processes by the civil construction industry. A growing possibility is the use of materials based on biopolymers, which are obtained by non-conventional transformation routes and are not derived from petroleum. In this scenario, the objective of the study was to identify the scientific production related to the biopolymers used in the construction industry through a bibliometric analysis for the period from 2010 to 2020. The data repository used was Scopus Elsevier, applying the filters according to the criteria of inclusion and exclusion. The results indicate that the publications are distributed in journals in the Engineering areas and also in those related to Environmental Sustainability and that the most cited authors, Ilhan Chang, Gye-Chun Cho and Jooyoung Im, were also the most productive and influential on the subject. The largest scientific contribution on the use of biopolymers in civil construction was given by the United States, followed by India and South Korea. It can be observed a significant increase in the number of publications from 2019, which demonstrates the importance that the community Since then, scientific research has given to the theme and also the distribution of publications among the different countries and research institutions, demonstrating the interest in this area in the various continents.
\end{abstract}

Keywords: Innovation; Raw material; Petroleum products; Socioenvironmental. 


\begin{abstract}
Resumen
Las demandas derivadas del contexto socioambiental sirven como estímulo para la innovación de productos y procesos por parte de la industria de la construcción civil. Una posibilidad creciente es el uso de materiales basados en biopolímeros, que se obtienen por vías de transformación no convencionales y no se derivan del petróleo. En este escenario, el objetivo del estudio fue identificar la producción científica relacionada con los biopolímeros utilizados en la industria de la construcción a través de un análisis bibliométrico para el período de 2010 a 2020 . El repositorio de datos utilizado fue Scopus Elsevier, aplicando los filtros según los criterios de inclusión y exclusión. Los resultados indican que las publicaciones se distribuyen en revistas de las áreas de Ingeniería y también en las relacionadas con Sostenibilidad Ambiental y que los autores más citados, Ilhan Chang, Gye-Chun Cho y Jooyoung Im, también fueron los más productivos e influyentes en el tema. El mayor aporte científico sobre el uso de biopolímeros en la construcción civil lo dio Estados Unidos, seguido de India y Corea del Sur. Se puede observar un aumento significativo en el número de publicaciones a partir de 2019, lo que demuestra la importancia que tiene la comunidad, la investigación científica ha dado al tema y también la distribución de publicaciones entre los diferentes países e instituciones de investigación, demostrando el interés por esta área en los distintos continentes.
\end{abstract}

Palabras clave: Innovación; Materias primas; Productos derivados del petróleo; Socioambiental.

\title{
1. Introdução
}

Atualmente, as empresas lidam com um cenário cada vez mais competitivo, onde muitas vezes a inovação é uma questão de sobrevivência, principalmente a inovação voltada aos produtos ambientalmente sustentáveis e economicamente viáveis. A conscientização do desenvolvimento empresarial que leve em consideração a sustentabilidade ambiental atingiu a realidade empresarial e tem feito com que tanto os consumidores quanto as empresas busquem alternativas que venham mitigar os impactos ambientais, tornando o meio produtivo mais eficiente e menos impactante ao ambiente (Medeiros et al., 2014). A inovação no desenvolvimento de produtos que respeite o compromisso com o meio ambiente em seus processos de produção vem ganhando cada vez mais importância entre as organizações, preocupadas com as questões ambientais e com iniciativas de sustentabilidade (Dangelico et al., 2017). O investimento ambiental tornou-se necessário, e a inovação verde é uma das estratégias importantes para se obter o desenvolvimento sustentável nas indústrias, podendo aumentar o valor do produto e compensando, dessa maneira, os custos dos investimentos voltados para o meio ambiente (Chang, 2011). O desenvolvimento de novos produtos pensando na preservação ambiental passou a ser uma questão estratégica para a competitividade entre as empresas (Lee \& Kim, 2012).

O crescimento industrial tem provocado sérios danos ambientais, o que tem levado a sociedade a discutir formas de reverter o quadro de degradação, que transcende fronteiras e afeta grandes extensões regionais. O mercado está numa busca cada vez maior por produtos que apresentem melhor visibilidade aos consumidores (Heinzen et al., 2011). As alterações climáticas e ambientais têm afetado de forma crescente o modo como as organizações definem seus planejamentos estratégicos e promovido a mudança de práticas em vários setores da economia. Como exemplo dessas mudanças temos a indústria da construção civil, com a utilização das práticas de Green Buildings, que possibilitam a aplicação dos princípios da química verde para o desenvolvimento de produtos ecologicamente corretos destinados à indústria da construção (Meirelles, 2014).

O conceito de Green Buildings evoluiu e vem ganhando impulso em todo o mundo, uma vez que incorpora considerações ambientais em todas as fases da construção visando, entre outras coisas, usar os recursos naturais de forma inteligente e reduzir impactos ambientais (Ojo-Fafore et al., 2018). De acordo com Almeida e Moraes (2011), as tecnologias emergentes moldarão o futuro de alguns setores, transformando muitos outros, determinando o surgimento e a reestruturação de indústrias em um ritmo sem precedentes, exigindo a revisão de marcos regulatórios e de modelos de negócio. Entre esses setores está o da Construção Civil, em que são previstas várias tecnologias emergentes e portadoras de futuro, como é o caso da química verde com o uso de matérias primas verdes na construção civil, os biopolímeros.

Os polímeros são considerados um recurso material bastante importante em quase todos os ramos da engenharia. Pesquisadores têm se empenhado ativamente na constante inovação de materiais poliméricos a serem utilizados na engenharia civil buscando diversificar seu uso e também aumentar sua resistência e durabilidade (James, 2019). A utilização de polímeros 
pela construção civil tem sido cada vez maior. Os polímeros possuem um vasto leque de aplicações e substituem materiais considerados nobres, como o aço, a madeira, o barro e o concreto. No entanto, a origem dos polímeros sintéticos ou artificiais, em geral, está no petróleo, uma fonte não renovável de matéria prima, o que faz com que exista uma grande preocupação com relação à sustentabilidade do ambiente construtivo (Hipolito et al., 2013).

$\mathrm{Na}$ engenharia civil existe uma preocupação contínua em encontrar soluções inovadoras e sustentáveis. O uso de materiais ecológicos no lugar de substâncias químicas tradicionais é um dos aspectos fundamentais. Assim, os biopolímeros são reconhecidos como uma alternativa em substituição aos polímeros químicos convencionais devido ao seu baixo impacto ambiental (Aminpour \& O'Kelly, 2015). Portanto, os biopolímeros vêm ganhando espaço, por serem facilmente degradados na natureza e também por serem produzidos a partir de fontes renováveis (Silva \& Rabelo, 2017). Segundo Vinod et al. (2020), a utilização de materiais de base biológica, que são renováveis no campo da construção civil, irá promover a sustentabilidade, reduzindo desperdícios, volume de aterros e emissões tóxicas, contribuindo para um ambiente mais verde e mais limpo.

Nos últimos anos foram realizados avanços significativos sobre o uso, desenvolvimento e aplicações de materiais poliméricos biodegradáveis preferidos por possuírem propriedades físicas, químicas, biológicas, biomecânicas e de degradação específicas (Hassan et al., 2019). Pesquisadores têm desenvolvido estudos contínuos sobre a substituição dos polímeros convencionais por biopolímeros que atendam às exigências das indústrias, uma vez que a ampla utilização de polímeros convencionais contribui enormemente para o aumento do volume de resíduos que agridem o meio ambiente, enquanto os biopolímeros possuem degradação mais rápida (Silva \& Rabelo, 2017).

Considerando que a introdução de inovações se torna cada vez mais importante para que as empresas possam atender às exigências do mercado, é necessário que as instituições sejam estimuladas a desenvolver atividades de ciência e tecnologia articuladas com as demandas do setor produtivo. Segundo Ferreira (2019), é importante que seja realizada uma análise sobre toda a produção científica gerada e que esse conhecimento se torne público, tanto sobre as atividades realizadas durante a pesquisa como sobre quais os resultados alcançados. Para isso, segundo Vanti (2002), a bibliometria é uma das formas de medição voltadas para avaliar a ciência e os fluxos de informação. Guedes e Borschiver (2005) destacam a aplicação da análise bibliométrica como ferramenta estatística básica para a gestão da informação e do conhecimento científico e tecnológico. Por meio dos estudos bibliométricos é possível coletar, organizar e processar informações que poderão ser utilizadas para orientar o desenvolvimento de novas pesquisas, promover inovação e fornecer base para a tomada de decisões (Miranda et al., 2019).

Nos últimos anos, os critérios cientométricos têm sido cada vez mais utilizados, a fim de avaliar a eficácia das instituições e seu impacto no desenvolvimento da ciência mundial (Khrustalev et al., 2019). As técnicas bibliométricas permitem realizar o mapeamento de informações e avaliar a produtividade da pesquisa científica, mediante o dimensionamento do volume de publicações e citações, possibilitando identificar o avanço tecnológico, o que poderá influir diretamente no planejamento de futuras ações a serem desenvolvidas (Machado. et al., 2016). A análise desses indicadores permite identificar tendências, que servirão de subsídio para a gestão dos processos de ciência, tecnologia e inovação e da informação para estratégias de tomada de decisão pelas organizações (Amador et al., 2018).

Destaca-se a relevância em se conhecer o panorama da produção científica relacionada às inovações e ao emprego de materiais a base de biopolímeros pela indústria da construção civil, identificando o estado da arte e avaliando a evolução e a contribuição do conhecimento científico sobre o tema em estudo. Nesse sentido esse artigo teve como objetivo identificar a produção científica sobre a utilização de materiais a base de biopolímeros pela indústria da construção civil através de uma análise bibliométrica referente ao período de 2010 a 2020. 


\section{Metodologia}

No presente estudo foi realizada uma pesquisa bibliográfica abrangente em uma das bases de dados on-line com posterior análise bibliométrica. Trata-se de uma pesquisa quali-quantitativa que, segundo Creswell (2010), consiste em uma abordagem investigativa que associa aspectos qualitativos e quantitativos, fazendo o uso das duas abordagens em comparação com a pesquisa qualitativa ou quantitativa isolada (Creswell, 2010).

De acordo com Xavier et al. (2012), a bibliometria corresponde a um conjunto de métodos matemáticos e estatísticos utilizados para investigar e quantificar a produção científica, que apresentam um conjunto de indicadores que possibilitam realizar uma interpretação do desempenho dos pesquisadores, instituições de pesquisa e países em determinado campo de pesquisa. Entre os diversos parâmetros passíveis de estudo estão autores, palavras-chave, citações, periódicos e publicações, ano de publicação, origem dos trabalhos, áreas do conhecimento, entre outros.

Em uma análise bibliométrica, a primeira fase, caracteriza-se pela escolha da base de dados, definição dos parâmetros de inclusão e exclusão de documentos e busca por documentos. A segunda fase, a fase de exploração, constitui-se pela administração das técnicas, ou seja, a bibliometria em si. E a terceira fase, de interpretação dos resultados, estabelece as inferências obtidas pelos resultados (Reynaud \& Todescat, 2017).

\subsection{Seleção dos documentos}

Os artigos que compõem a amostra deste estudo foram obtidos através da base de dados eletrônica Scopus®, que contém resumos e citações da literatura revisados às cegas por pares: revistas científicas, livros, processos de congressos e publicações do setor. Esse banco de dados oferece um panorama abrangente da produção de pesquisas do mundo todo nas áreas de ciência, tecnologia, medicina, ciências sociais, artes e humanidades e disponibiliza ferramentas inteligentes para monitorar, analisar e visualizar pesquisas. A base de dados Scopus disponibiliza também elementos para análise de citações, referências, entre outras (Elsevier, 2020). Este banco de dados foi escolhido em função de armazenar um número considerável de documentos amplamente reconhecidos cientificamente, em diferentes áreas.

$\mathrm{Na}$ presente revisão bibliométrica foram considerados, como critérios para inclusão e exclusão, somente estudos primários, artigos e revisões, referentes às áreas "Engineering" e "Environmental Science". Foram excluídos os estudos referentes às demais áreas. A busca foi feita em documentos no idioma inglês. Estudos em outros idiomas foram excluídos, uma vez que a maioria dos artigos publicados está em inglês.

Para seleção de dados da amostra, primeiramente foi realizada uma leitura de todos os títulos e resumos de cada documento encontrado, com o objetivo de excluir aqueles documentos que não apresentavam relação com o tema selecionado. Procedeu-se a uma análise detalhada de cada resumo, baseada nos critérios de inclusão e exclusão adotados e no tema norteador do presente estudo. Foram, então, excluídos os documentos identificados como aqueles que não correspondiam ao objetivo da presente revisão.

A partir daí foi realizada uma leitura de todos os artigos na íntegra, quando se decidiu pela inclusão ou exclusão dos documentos previamente selecionados.

\subsection{Análise dos dados}

Para análise dos dados foi utilizado o aplicativo Biblioshiny, que é um pacote desenvolvido para a linguagem R que fornece um conjunto de ferramentas para pesquisas relacionadas à cientometria e à bibliometria e que permite avaliar três grandes grupos: a) autores, incluindo análises relacionadas à autoria, à filiação e aos países; b) fontes de publicações, 
permitindo a avaliação do impacto das fontes e verificação da produtividade; e c) documentos, englobando referências e palavras mais citadas (Aria \& Cuccurullo, 2017).

Foi também utilizado o software VOSviewer que é uma ferramenta para construção e visualização de redes bibliométricas, incluindo periódicos, pesquisadores e publicações individuais. As redes bibliométricas são construídas com base em citações, acoplamentos bibliográficos, cocitações ou relações de coautoria. Em uma análise de cocitação dos pesquisadores, a relação dos pesquisadores é determinada com base no grau em que são citados nas mesmas publicações (VOSviewer, 2020). Quanto mais frequentemente dois pesquisadores são citados nas mesmas publicações, mais forte é a relação (Perianes-Rodriguez et al., 2016). O VOSviewer também oferece a funcionalidade de mineração de texto, que pode ser usada para construir e visualizar redes de ocorrência simultânea de termos importantes extraídos de um corpo de literatura científica (VOSviewer, 2020).

\section{Resultados e Discussão}

Para uma análise mais completa é necessário que, além de quantificar as publicações de uma instituição de pesquisas de determinada área ou país, se faça comparações entre esses dados e o monitoramento da produção científica ao longo do tempo (Bordons \& Zulueta, 1999).

Após ser definida a plataforma que seria utilizada na pesquisa e os critérios de busca e análise de dados, foram definidas algumas palavras-chave que apresentavam relação com a temática deste estudo. A busca foi realizada utilizando os descritores "biopolymer", "building", "construction" e "green polymer" e os conectores "OR" e "AND", nos campos do título, resumo e palavras-chave das publicações, no período de estudo limitado de 2010 a 2020.

A expressão de busca obtida foi: TITLE-ABS-KEY ( ( biopolymer OR "green polymer" ) AND ( construction OR building)) AND DOCTYPE ( ar OR re) AND PUBYEAR > 1999 AND PUBYEAR < 2020 AND (LIMIT-TO ( SUBJAREA, "ENGI" ) OR LIMIT-TO ( SUBJAREA, "ENVI" ))

$\mathrm{Na}$ busca inicial foram recuperadas 334 publicações que foram analisadas fazendo-se a leitura do título e resumo das mesmas. Foram excluídos aqueles documentos que não apresentaram relação com o tema em estudo ou que não estavam dentro dos parâmetros de pesquisa desejados, resultando em uma amostra de 57 publicações para o presente estudo.

Observando-se a Figura 1 é possível verificar a produção científica anual sobre o uso de biopolímeros na construção civil, no período de 2000 a 2020. 
Figura 1 - Produção científica anual sobre o uso de biopolímeros no período de 2001 a 2020.

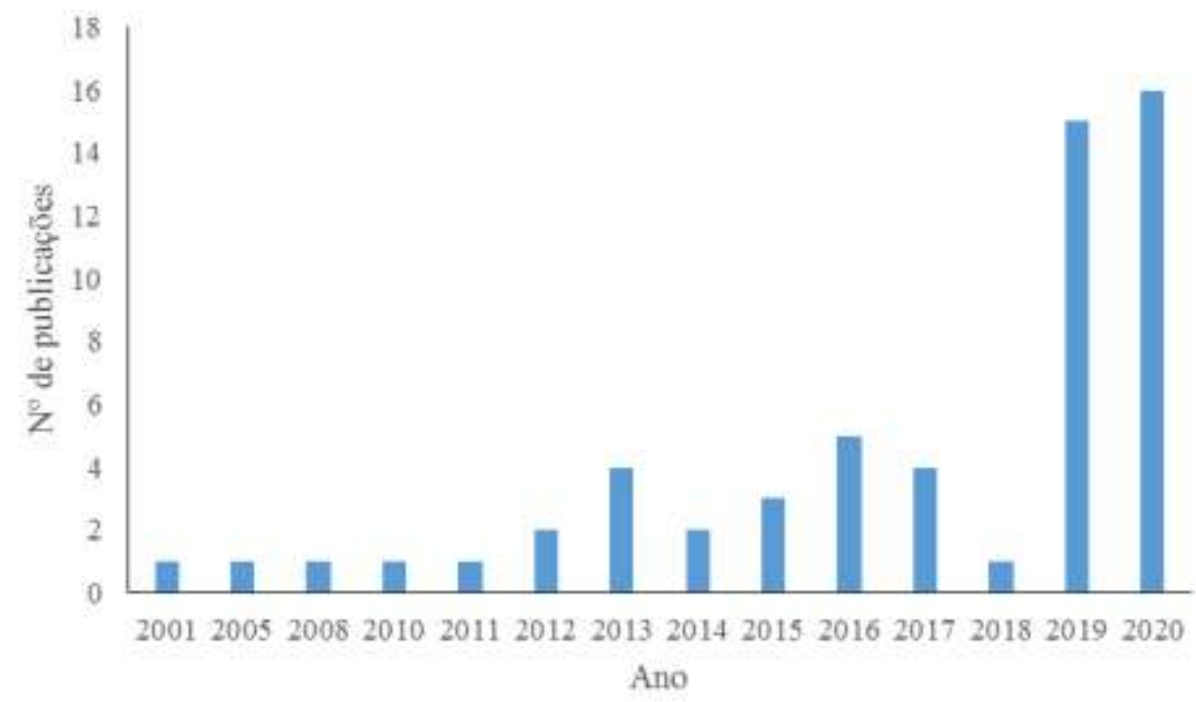

Fonte: Autores, com base nos dados da Scopus® (2020).

A produção científica, desde o início do período de estudo até o ano de 2018, é bastante incipiente com publicação de menos de cinco artigos por ano, excetuando-se apenas o ano de 2016 em que foram publicados exatos cinco artigos. Porém, observa-se um aumento significativo no número de artigos publicados a partir de 2019, o que evidencia que o interesse da comunidade científica pelo tema em questão ganhou bastante ênfase a partir deste ano, provavelmente devido ao surgimento das novas demandas que levam em conta a questão ambiental.

A crescente preocupação com a questão ambiental e a descoberta dos impactos ambientais ocasionados pelo uso de materiais derivados da indústria petroquímica impulsionaram a busca por alternativas sustentáveis. Entre essas alternativas, em substituição aos polímeros, também chamados termoplásticos e termorrígidos, estão os biopolímeros, polímeros naturais oriundos de recursos naturais renováveis, de degradação mais rápida em relação aos materiais derivados do petróleo no meio ambiente (Farias et al., 2016). Os plásticos fabricados a partir de derivados do petróleo podem ser substituídos por polímeros de base biológica, oriundos de recursos de biomassa, disponíveis de forma renovável e recorrente, e que mantêm as características de biodegradabilidade e renovabilidade (Thakur et al., 2018).

Vários países vêm pesquisando sobre o uso de biopolímeros pela indústria da construção civil, destacando-se pelo maior número de publicações com destaque para dez países (Figura 2). 
Figura 2 - Frequência de participação na produção científica por país.

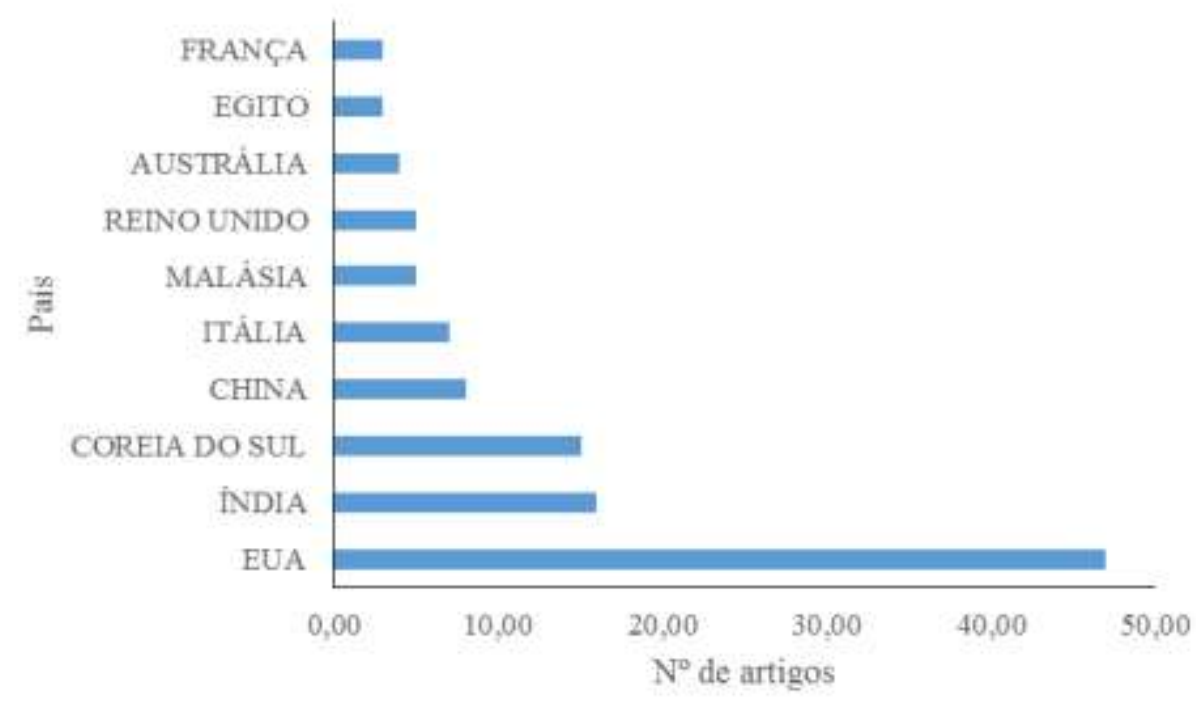

Fonte: Autores, com base nos dados da Scopus ${ }^{\circledR}$ (2020).

A Figura 2 indica a frequência de participação de cada país na produção científica mundial sobre o tema em questão. Destaca-se a participação dos Estados Unidos (47,0 \%), seguido pela Índia (16,0 \%) e Coréia do Sul (15,0 \%). O Brasil não compõe o grupo dos dez países com produção científica nessa área.

Em 2012, o governo americano, por meio do "National Bioeconomy Blueprint", estabeleceu objetivos estratégicos para alcançar todo o potencial da bioeconomia nos EUA, prevendo a inclusão de novos materiais no mercado, incluindo plásticos biodegradáveis derivados de biomassa renovável (White House, 2012). Segundo Castelan e Pinotti (2019), a Índia se caracteriza como uma economia emergente e com rápido crescimento econômico e, por isso, vem investindo em pesquisas relacionadas principalmente à sustentabilidade e questões ambientais, com o objetivo de amenizar os efeitos do desenvolvimento. A Coreia do Sul também tem investido em educação voltada para a produção do conhecimento e a inovação tecnológica (Zagato, 2019).

A Figura 3 indica os dez periódicos científicos que mais publicaram sobre o tema e a respectiva quantidade de artigos publicados. 
Figura 3 - Periódicos e quantidade de artigos publicados.

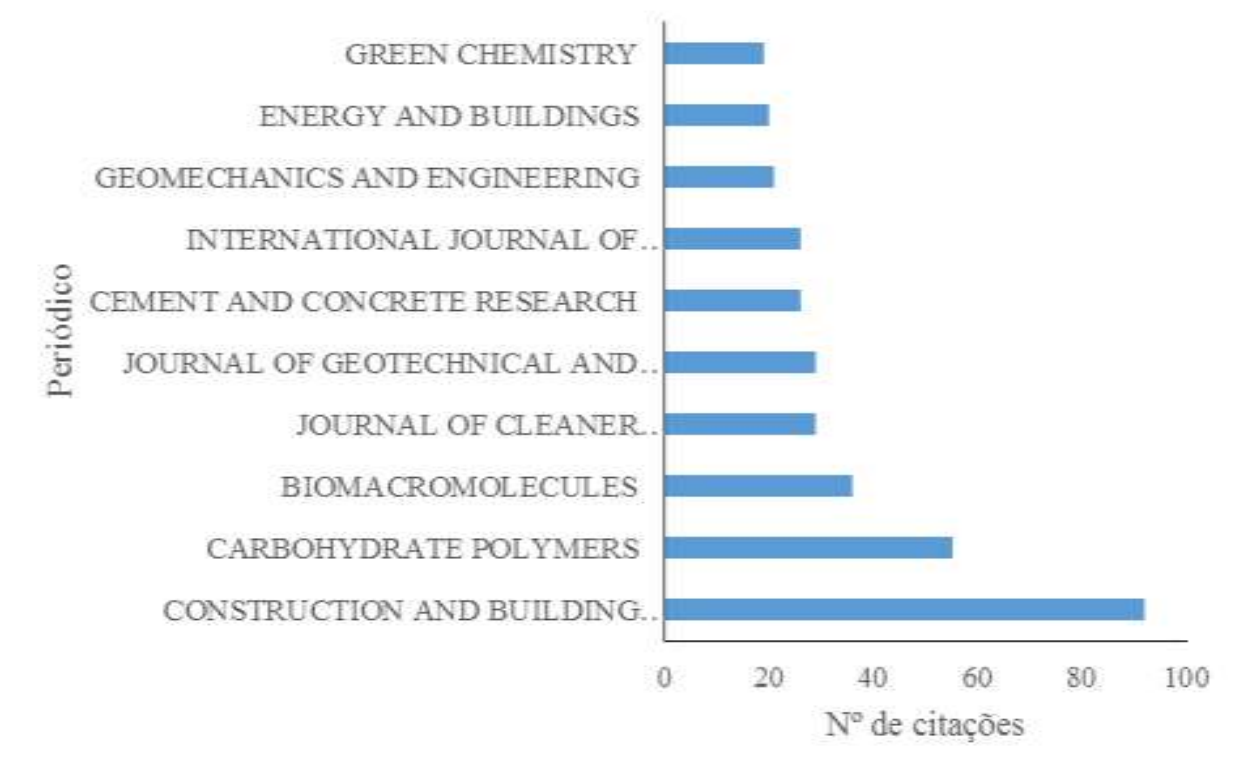

Fonte: Autores, com base nos dados da Scopus® (2020).

Esses periódicos são responsáveis pela publicação de 45,61 \% dos artigos levantados na amostra. Os periódicos destacados atendem à definição da Lei de Bradford, que estabelece ser possível identificar o grupo de periódicos que publicam o maior número de artigos sobre o tema, formando um núcleo de periódicos de maior relevância para determinada área (Araújo, 2006). Nesse estudo o núcleo mencionado é composto pelos periódicos: Construction and Building Materials, Journal of Cleaner Production, Sustainability (Switzerland), Composites Part B: Engineering, Journal of Materials in Civil Engineerin, Journal of Polymers and the Environment; ACS Sustainable Chemistry and Engineering; Advanced Materials, Advanced Sustainable Systems e Advances in Civil Engineering, Observa-se que as publicações sobre o tema se concentram não só em periódicos relacionados especificamente à construção civil, mas também naqueles relacionados à sustentabilidade ambiental.

Dos 195 autores que publicaram artigos científicos sobre a temática, identificados na amostra, aqueles que tiveram maior participação na produção científica são apresentados na Figura 4. 
Figura 4 - Participação dos autores na produção científica.

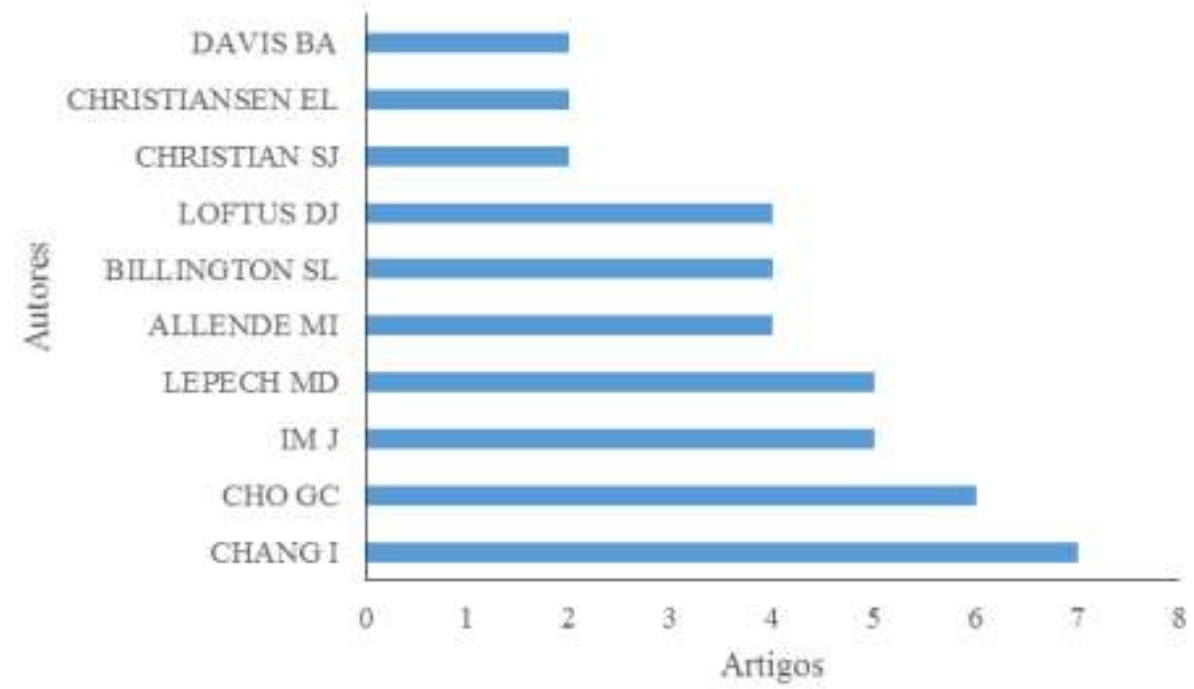

Fonte: Autores, com base nos dados da Scopus ${ }^{\circledR}$ (2020).

Os autores apresentados na Figura 4 colaboraram com 72,0 \% de toda produção sobre o tema. Os demais autores que contribuíram com um ou dois artigos publicados sobre o assunto, foram contabilizados, mas não são apresentados na figura. Este indicador atende à Lei de Lotka que, segundo Café e Bräscher (2008), visa identificar os pesquisadores que mais contribuem em determinada área do conhecimento. Para Lotka (1926), em várias áreas do conhecimento existem poucos pesquisadores com muita produção científica e muitos pesquisadores com baixa produção.

Segundo Bordons e Zulueta (1999), o número de citações referentes a determinado autor é um indicador utilizado para medir a influência desse autor sobre a comunidade científica de determinada área, que irá referenciá-los em trabalhos subsequentes. Assim, a Figura 5 apresenta os autores mais citados. Sendo que ganham destaque os autores Ilhan Chang, GyeChun Cho e Jooyoung Im, que estão também entre os pesquisadores que mais publicaram.

Figura 5 - Autores mais citados nas publicações.

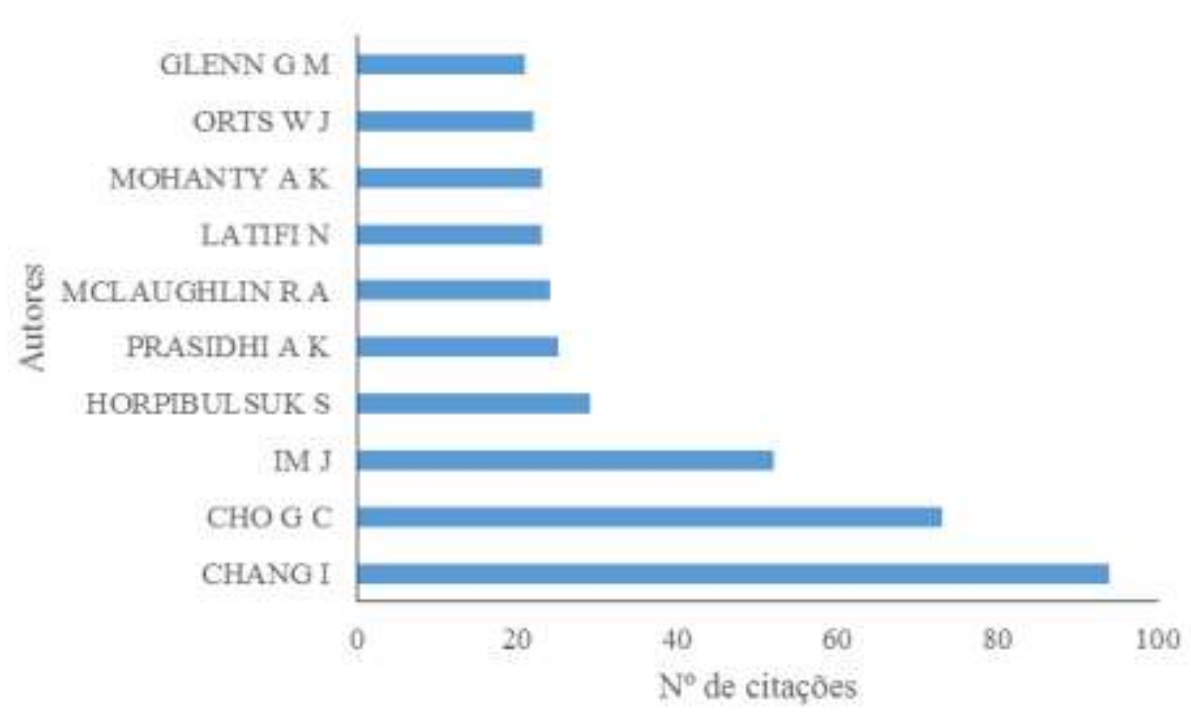

Fonte: Autores, com base nos dados da Scopus ${ }^{\circledR}(2020)$. 
Para uma análise mais abrangente e com o objetivo de conhecer a relação entre os autores mais citados, foi construída a Tabela 01, que apresenta os autores mais citados e as instituições de pesquisa que representavam na época da publicação.

Tabela 1 - Autores que mais contribuíram com a produção científica e instituições de pesquisa que representam.

\begin{tabular}{l|l}
\multicolumn{1}{c|}{$\begin{array}{c}\text { Autores que mais } \\
\text { produziram }\end{array}$} & \multicolumn{1}{c}{ Instituição de pesquisa } \\
\hline Ilhan Chang & $\begin{array}{l}\text { SOC Research Institute, Korea Institute of Civil Engineering and Building } \\
\text { Technology (KICT), Goyang 411-712, Republic of Korea }\end{array}$ \\
\hline Gye-Chun Cho & $\begin{array}{l}\text { Dept. Civil and Environmental Eng., Korea Advanced Institute of Science } \\
\text { and Technology (KAIST), Daejeon 305-701, Republic of Korea }\end{array}$ \\
\hline Jooyoung Im & $\begin{array}{l}\text { Dept. Civil and Environmental Eng., Korea Advanced Institute of Science } \\
\text { and Technology (KAIST), Daejeon 305-701, Republic of Korea }\end{array}$ \\
\hline Michael D. Lepech & $\begin{array}{l}\text { Department of Civil and Environmental Engineering, Stanford University, } \\
\text { 473 Via Ortega, Stanford, CA 94305-4020, USA }\end{array}$ \\
\hline Maria I. Allende & $\begin{array}{l}\text { Department of Civil and Environmental Engineering, Stanford University, } \\
\text { Stanford, CA 94305, United States }\end{array}$ \\
\hline S L Billington & $\begin{array}{l}\text { Department of Civil and Environmental Engineering, Stanford University, } \\
\text { Stanford, CA 94305-4020, United States }\end{array}$ \\
\hline David J. Loftus & $\begin{array}{l}\text { Division of Space Biosciences, NASA Ames Research Center, Moffet Field, } \\
\text { CA 94035, USA }\end{array}$ \\
\hline S.J. Christian & $\begin{array}{l}\text { Department of Civil and Environmental Engineering, Carnegie Mellon } \\
\text { University, Pittsburgh, PA 15213-3890, United States }\end{array}$ \\
\hline Eric L. Christiansen & $\begin{array}{l}\text { Astromaterials Research and Exploration Science, NASA Johnson Space } \\
\text { Center, Houston, TX 77058, United States }\end{array}$ \\
\hline B. Alan Davis & $\begin{array}{l}\text { Jacobs Technologies, NASA Johnson Space Center, Houston, TX 77058, } \\
\text { United States }\end{array}$ \\
\hline
\end{tabular}

Fonte: Autores, com base nos dados da Scopus® (2020).

A Tabela 2 apresenta as publicações dos autores mais citados, que se encontram dentro da amostra em questão, bem como os países onde estão localizadas as instituições de pesquisa que representavam na época da publicação.

Tabela 2 - Publicações encontradas na amostra dos autores mais citados e países das instituições de pesquisa

\begin{tabular}{|c|c|c|}
\hline $\begin{array}{l}\text { AUTORES QUE MAIS } \\
\text { PRODUZIRAM }\end{array}$ & $\begin{array}{c}\text { TÍTULO DOS ARTIGOS DA } \\
\text { AMOSTRA } \\
\end{array}$ & PAÍS \\
\hline $\begin{array}{l}\text { Ilhan Chang } \\
\text { Gye-Chun Cho } \\
\text { Jooyoung Im }\end{array}$ & $\begin{array}{l}\text { Effects of Xanthan gum biopolymer on } \\
\text { soil strengthening }\end{array}$ & Coreia do Sul \\
\hline $\begin{array}{l}\text { Ilhan Chang } \\
\text { Gye-Chun Cho } \\
\text { Jooyoung Im } \\
\end{array}$ & $\begin{array}{l}\text { Soil strengthening using thermo- } \\
\text { gelation biopolymers }\end{array}$ & Coreia do Sul \\
\hline $\begin{array}{l}\text { Ilhan Chang } \\
\text { Gye-Chun Cho } \\
\text { Jooyoung Im }\end{array}$ & $\begin{array}{l}\text { Introduction of Microbial Biopolymers } \\
\text { in Soil } \\
\text { Treatment for Future Environmentally- } \\
\text { Friendly and Sustainable Geotechnical } \\
\text { Engineering }\end{array}$ & Coreia do Sul \\
\hline $\begin{array}{l}\text { Ilhan Chang } \\
\text { Gye-Chun Cho }\end{array}$ & $\begin{array}{l}\text { Geotechnical engineering behavior of } \\
\text { biopolymer-treated soft marine soil }\end{array}$ & Coreia do Sul \\
\hline Ilhan Chang & $\begin{array}{l}\text { Xanthan Gum Biopolymer as Soil- } \\
\text { Stabilization Binder for Road } \\
\text { Construction Using Local Soil in Sri } \\
\text { Lanka }\end{array}$ & Coreia do Sul \\
\hline $\begin{array}{l}\text { Michael D. Lepech } \\
\text { SL Billington }\end{array}$ & $\begin{array}{l}\text { Influence of carbon feedstock on } \\
\text { potentially net beneficial } \\
\text { environmental impacts of bio-based }\end{array}$ & Estados Unidos \\
\hline
\end{tabular}


Research, Society and Development, v. 10, n. 4, e17210414025, 2021

(CC BY 4.0) | ISSN 2525-3409 | DOI: http://dx.doi.org/10.33448/rsd-v10i4.14025

\begin{tabular}{|c|c|c|}
\hline & composites & \\
\hline $\begin{array}{l}\text { Michael D. Lepech } \\
\text { Maria I. Allende } \\
\text { David J. Loftus }\end{array}$ & $\begin{array}{l}\text { On Designing Biopolymer-Bound Soil } \\
\text { Composites (BSC) for Peak } \\
\text { Compressive Strength }\end{array}$ & Estados Unidos \\
\hline $\begin{array}{l}\text { Michael D. Lepech } \\
\text { Maria I. Allende } \\
\text { David J. Loftus } \\
\text { Christiansen EL } \\
\text { Davis BA }\end{array}$ & $\begin{array}{l}\text { Hypervelocity Impact Performance of } \\
\text { Biopolymer-Bound Soil Composites for } \\
\text { Space Construction } \\
\text { Prediction of micrometeoroid damage } \\
\text { to lunar construction materials using } \\
\text { numerical modeling of hypervelocity } \\
\text { impact events }\end{array}$ & Estados Unidos \\
\hline $\begin{array}{l}\text { S L Billington } \\
\text { S.J. Christian }\end{array}$ & $\begin{array}{l}\text { Mechanical response of } \mathrm{PHB} \text { - and } \\
\text { cellulose acetate natural fiber- } \\
\text { reinforced } \\
\text { composites for construction } \\
\text { applications } \\
\text { Characterization of poly- } \\
\text { hydroxybutyrate films and hemp fiber } \\
\text { reinforced } \\
\text { composites exposed to accelerated } \\
\text { weathering }\end{array}$ & Estados Unidos \\
\hline
\end{tabular}

Fonte: Autores, com base nos dados da Scopus ${ }^{\circledR}(2020)$.

Analisando-se a Tabela 1 e a Tabela 2, foi identificada a interação entre diferentes instituições de pesquisa localizadas na Coreia do Sul e representadas por Ilhan Chang (SOC Research Institute, Korea Institute of Civil Engineering and Building Technology (KICT)), Gye-Chun Cho e Jooyoung Im (Dept. Civil and Environmental Eng., Korea Advanced Institute of Science and Technology $(K A I S T))$. Ocorre interação também entre as instituições de pesquisa localizadas nos Estados Unidos e representadas por Michael D. Lepech e Maria I. Allende (Department of Civil and Environmental Engineering, Stanford University) e David J. Loftus (Division of Space Biosciences, NASA Ames Research Center) e entre diferentes departamentos pertencentes à NASA, representados por David J. Loftus (Division of Space Biosciences, NASA Ames Research Center), Christiansen EL (Astromaterials Research and Exploration Science, NASA Johnson Space Center) e Davis BA (Jacobs Technologies, NASA Johnson Space Center) e entre a instituição representada por SL Billington (Department of Civil and Environmental Engineering, Stanford University, Stanford) e S.J. Christian (Department of Civil and Environmental Engineering, Carnegie Mellon University, Pittsburgh).

As coautorias podem ser identificadas por meio da Figura 6 elaborada a partir do uso do VOSviewer. 
Figura 6 - Coautoria nas publicações apresentadas pelo VOSviewer.

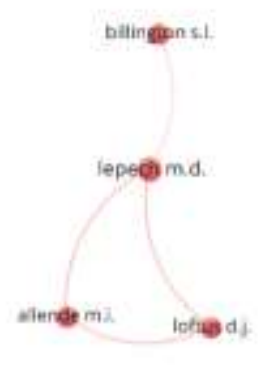

Fonte: Autores, com base nos dados da Scopus ${ }^{\circledR}(2020)$.

$\mathrm{Na}$ Tabela 2 podemos identificar que os pesquisadores que mais contribuíram com a produção científica do presente estudo desenvolveram suas pesquisas em conjunto, sobre a utilização de material polimérico para melhoria das propriedades dos solos, como estabilidade do agregado, força e resistência à erosão e sobre compósitos de base biológica.

Outros autores menos citados também realizaram estudos sobre o uso de biopolímeros para estabilização e melhoria das propriedades físicas do solo.

Além dos trabalhos já citados, também foi identificada produção científica sobre:

- Substituição de agregado fino por material a base de biopolímeros no concreto utilizado para produção de telhas;

- Utilização de biopolímeros na composição de revestimentos das superfícies das edificações;

- Biopolímeros como floculante no controle da turbidez da água proveniente do bombeamento das escavações em locais de construção;

- Melhorias nas propriedades físicas e mecânicas do concreto endurecido com a adição de biopolímero de goma arábica à mistura;

- Aplicação de biopolímeros a base de amido de milho na produção de uma nova construção material, denominado CoRncrete;

- O desenvolvimento de material de isolamento térmico composto por biopolímeros;

- Melhoria do desempenho de mistura asfálticas com a introdução de biopolímeros;

- Na composição de plastificante na fabricação de concreto;

- Outros. 
Figura 7 - Apresenta os dez países com maior número de citações bibliográficas.

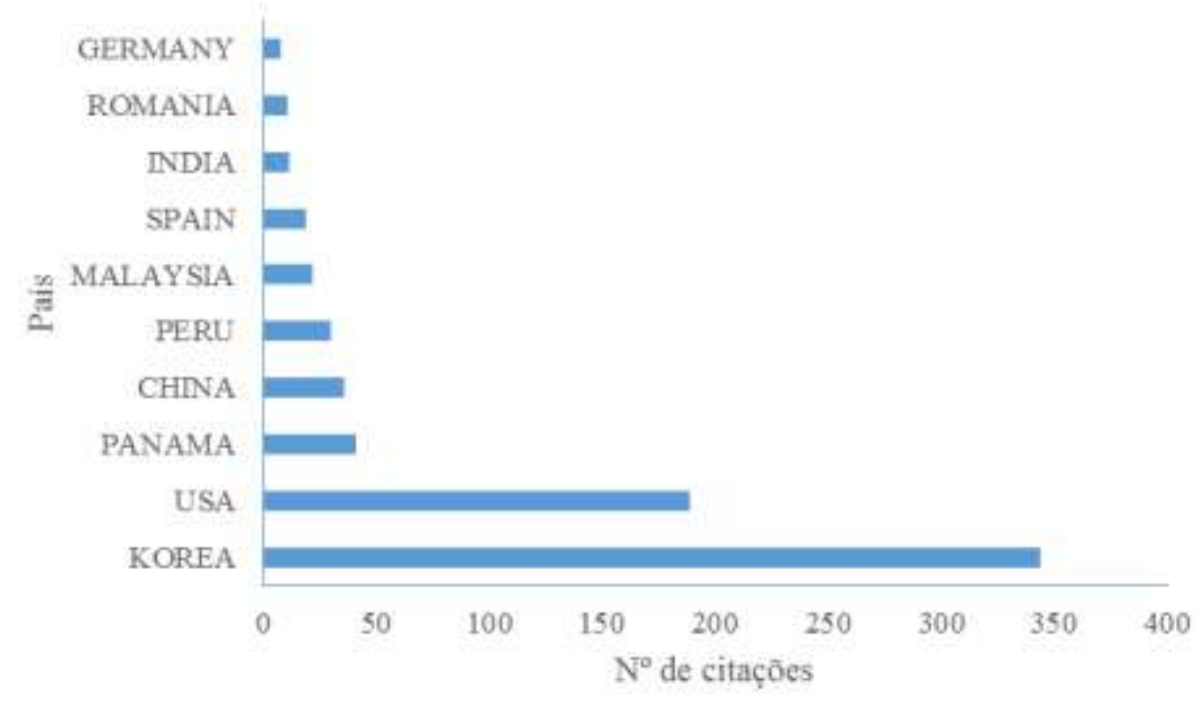

Fonte: Autores, com base nos dados da Scopus ${ }^{\circledR}$ (2020).

De acordo com a Figura 7, a Coreia do Sul aparece como o país com o maior número de citações, equivalente a 47,1 \% do total de citações levantadas, seguida pelo Estados Unidos, com 25,9 \%, o que está de acordo com os dados levantados anteriormente sobre a participação na produção científica por país, participação dos autores na produção científica e autores mais citados.

Segundo Meseguer-Sánchez et al. (2020) as instituições de pesquisa, além da educação para a sustentabilidade, promovem um grande impacto na sociedade e no desenvolvimento econômico, ambiental e social, por meio do ensino superior, pesquisa e transferência de conhecimento e tecnologia.

A Figura 8 mostra a participação na produção científica das dez instituições de pesquisa que mais têm contribuído com o tema, o que corresponde a $23,9 \%$ da participação de todas as instituições levantadas.

Figura 8 - Produção das instituições de pesquisa.

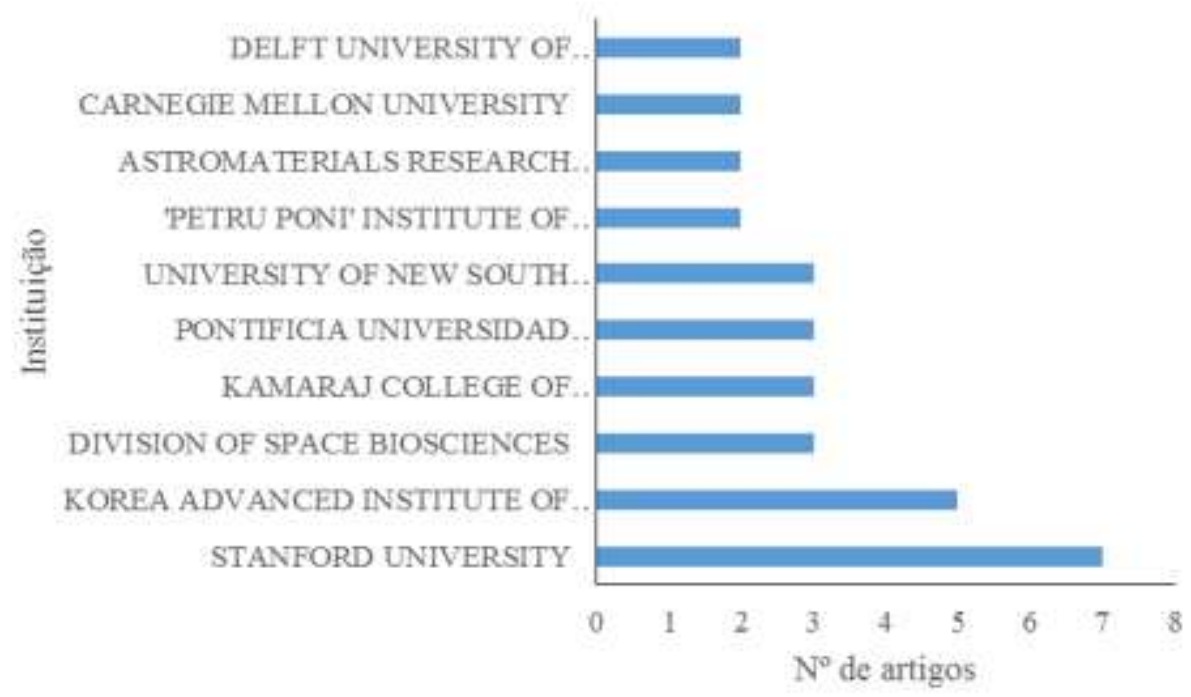

Fonte: Autores, com base nos dados da Scopus ${ }^{\circledR}$ (2020). 
A Stanford University (EUA) responde por 5,2 \% do total da participação na produção científica de todas as instituições, Korea Advanced Institute of Science and Technology - KAIST (Coréia do Sul) por 3,7 \%, e Division of Space Biosciences- NASA (Estados Unidos), Kamaraj College of Engineering and Technology (Índia), Pontificia Universidad Catlica Del Per Pucp (Peru) e University of New South Wales (Austrália) por 2,2\%.

Apesar da Índia aparecer em oitavo lugar quando se refere aos dez países com maior número de citações, a Kamaraj College of Engineering and Technology aparece entre as instituições que mais têm contribuído e que estão posicionadas em terceiro lugar.

\section{Considerações Finais}

Através da análise bibliométrica realizada, referente ao período de 2010 a 2020, foi possível investigar os autores e publicações que tiveram relação com o estudo sobre biopolímeros e seu uso na construção civil, observando-se a relevância do assunto em estudo em diversos países e áreas de pesquisa dentro da engenharia civil.

Foram analisadas informações relacionadas à distribuição anual de publicações, principais periódicos, autores que mais publicaram e aqueles mais citados, países e instituições de pesquisa que mais contribuíram com a produção científica sobre o tema em estudo.

Os resultados do estudo apontam para uma incipiente produção científica sobre o tema ao longo do período de estudo, porém, observa-se um aumento significativo desta produção no ano de 2019, o que demonstra a importância que a comunidade científica passou a dar ao tema a partir de então.

As publicações estão distribuídas em periódicos das áreas de Engenharia e também daqueles relacionados à Sustentabilidade Ambiental, e os autores mais citados, Ilhan Chang, Gye-Chun Cho e Jooyoung Im, foram também os mais produtivos e influentes sobre o tema.

A maior contribuição científica sobre o uso de biopolímeros na construção civil foi possibilitada pelos Estados Unidos, seguido por Índia e Coréia do Sul, e entre as principais instituições que conduziram pesquisas sobre o assunto estão a Stanford University (EUA), Korea Advanced Institute of Science and Technology - KAIST (Coréia do Sul), Division of Space Biosciences- NASA (Estados Unidos), Kamaraj College of Engineering and Technology (India), Pontificia Universidad Catlica Del Per Pucp (Peru) e University of New South Wales (Austrália).

Observou-se a distribuição das publicações sobre o tema entre os diversos países e instituições de pesquisa, demonstrando o interesse por essa área nos diversos continentes.

Recomenda-se que sejam conduzidas futuras avaliações bibliométricas e também patentométricas, tornando possível verificar como a produção científica sobre o tema estudado se comportará a partir de 2020 e também identificar as inovações e o desenvolvimento tecnológico nas diversas instituições e países.

\section{Referências}

Almeida, M. F. L. D., \& Moraes, C. A. C. D. (2012). Indústrias do futuro e tecnologias emergentes: visão de um futuro sustentável. Parcerias Estratégicas, 16(33), 135-162.

Amador, S. R., Pérez, M. D., López-Huertas, M. J., \& Font, R. J. R. (2018). Indicator system for managing science, technology and innovation in universities. Scientometrics, 115(3), 1575-1587.

Aminpour, M., \& O'Kelly, B. C. (2015). Applications of biopolymers in dam construction and operation activities. In Proceedings of the 2nd International Dam World Conference, Lisbon, Portugal. 1, 937-946. Laboratório Nacional de Engenharia Civil, Lisbon, Portugal.

Araújo, C. A. (2006). Bibliometria: evolução histórica e questões atuais. Em questão, 12(1), 11-32.

Bordons, M. (1999). Evaluación de la actividad científica a través de indicadores bibliométricos. Revista española de cardiología, 52(10), 790-800. 
Café, L., \& Bräscher, M. (2008). Organização da informação e bibliometria. Encontros Bibli: revista eletrônica de biblioteconomia e ciência da informação, (Esp), 54-75.

Catelan, T. C., \& Pinotti, L. M. (2019). Avanço das pesquisas envolvendo Aspergillus niger e bagaço da cana-de-açúcar como fonte de carbono visando à produção de celulases: Uma análise bibliométrica. Matéria (Rio de Janeiro), 24(2).

Chang, C. H. (2011). The influence of corporate environmental ethics on competitive advantage: The mediation role of green innovation. Journal of Business Ethics, 104(3), 361-370.

Creswell, J. W. (2010). Projeto de pesquisa métodos qualitativo, quantitativo e misto. In Projeto de pesquisa métodos qualitativo, quantitativo e mist (pp. 296296).

Dangelico, R. M., Pujari, D., \& Pontrandolfo, P. (2017). Green product innovation in manufacturing firms: A sustainability-oriented dynamic capability perspective. Business strategy and the Environment, 26(4), 490-506.

Elsevier. Sobre a solução Scopus Recuperado em 30 novembro, 2020, de https://www.elsevier.com/pt-br/solutions/scopus.

Farias, S. S., Siqueira, S. M. C., Cristino, J. H. S., \& da Rocha, J. M. (2016). Biopolímeros: uma alternativa para promoção do desenvolvimento sustentável. Revista Geonorte, 7(26), 61-77.

Ferreira, A. G. C. (2010). Bibliometria na avaliação de periódicos científicos. Revista de Ciência da Informação, 11(3), 1-9.

Guedes, V. L., \& Borschiver, S. (2005). Bibliometria: uma ferramenta estatística para a gestão da informação e do conhecimento, em sistemas de informação, de comunicação e de avaliação científica e tecnológica. Anais do Encontro Nacional de Ciência da Informação, 6(1), 18.

Hassan, M. E. S., Bai, J., \& Dou, D. Q. (2019). Biopolymers; definition, classification and applications. Egyptian Journal of Chemistry, 62(9), $1725-1737$.

Heinzen, D. de M., A., Mattos, A. P. M. N., de Souza Campos, L. M., \& Paladini, E. P. (2011). Estudo da viabilidade de produto inovador "verde" para o mercado consumidor comum. Revista Gestão Organizacional, 4(2), 122-131.

Hipolito, I. da S., Hipolito, R. da S, \& Lopes, G. de A. (2013). Polímeros na construção civil. Proceedings of the Simpósio de Excelencia em Gestão e Tecnologia, Resende, Brazil, 23-25.

James, M. B. (2019). Polymers in civil engineering: review of alternative materials for superior performance. Journal of Applied Science and Computations, $6(5)$.

Machado, C., Souza, M. T. S., dos Santos Parisotto, I. R., \& Palmisano, A. (2016). The laws of bibliometrics in different scientific databases. Revista de Ciências da Administração, 18(44), 111-123.

Khrustalev, M. B., Tishkov, A. V., Turbina, N. Y., \& Maksimova, A. A. (2019). Scientometric indices of russian centers active in oncology/hematology research. Cellular Therapy and Transplantation, 8(4), 8-14.

Lee, K. H., \& Kim, J. W. (2012). Green new product development and supplier involvement: strategic partnership for green innovation. International Journal of Innovation and Sustainable Development, 6(3), 290-304.

Lotka, A. J. (1926). The frequency distribution of scientific productivity. Journal of the Washington academy of sciences, 16(12), 317-323.

Medeiros, J. F., Ribeiro, J. L. D., \& Cortimiglia, M. N. (2014). Success factors for environmentally sustainable product innovation: a systematic literature review. Journal of Cleaner Production, 65, 76-86.

Meirelles, S. L. (2014). Química verde: a indústria química e seus impactos na indústria da construção. Anais do X Congresso Nacional de Excelência em Gestão. Rio de Janeiro, RJ, Brasil.

Meseguer-Sánchez, V., Abad-Segura, E., Belmonte-Ureña, L. J., \& Molina-Moreno, V. (2020). Examining the research evolution on the socio-economic and environmental dimensions on university social responsibility. International Journal of Environmental Research and Public Health, $17(13), 4729$.

Miranda, D. M. V. D., Dutra, L. D. S., Way, D., Amaral, N., Wegenast, F., Scaldaferri, M. C., \& Pinto, J. C. (2019). A bibliometric survey of Paraffin/Olefin separation using membranes. Membranes, 9(12), 157.

Ojo-Fafore, E., Aigbavboa, C., \& Remaru, P. (2018). Benefits of green buildings. In International Conference on Industrial Engineering and Operations Management (Vol. 1, p. 2289).

Perianes-Rodriguez, A., Waltman, L., \& Van Eck, N. J. (2016). Constructing bibliometric networks: A comparison between full and fractional counting. Journal of Informetrics, 10(4), 1178-1195.

Reynaud, P. D., \& Todescat, M. (2017). Avaliação de desempenho humano na esfera pública: Estado da arte na literatura internacional e nacional. REGERevista de Gestão, 24(1), 85-96.

Silva, F. A., \& Rabelo, D. (2017). O Uso Sustentável de Polímeros. Revista Processos Químicos, 11(21), 9-16.

Thakur, S., Chaudhary, J., Sharma, B., Verma, A., Tamulevicius, S., \& Thakur, V. K. (2018). Sustainability of bioplastics: Opportunities and challenges. Current opinion in Green and Sustainable chemistry, 13, 68-75.

Vanti, N. A. P. (2002). Da bibliometria à webometria: uma exploração conceitual dos mecanismos utilizados para medir o registro da informação e a difusão do conhecimento. Ciência da informação, 31(2), 369-379. 
Research, Society and Development, v. 10, n. 4, e17210414025, 2021

(CC BY 4.0) | ISSN 2525-3409 | DOI: http://dx.doi.org/10.33448/rsd-v10i4.14025

Vinod, A., Sanjay, M. R., Suchart, S., \& Jyotishkumar, P. (2020). Renewable and sustainable biobased materials: An assessment on biofibers, biofilms, biopolymers and biocomposites. Journal of Cleaner Production, 258, 120978.

VOSviewer (2020). Visualizing scientific landscapes. https://www.vosviewer.com/.

White House (2012). National Bioeconomy Blueprint. White House, 01-43.

Xavier, B. M., da Silva, A. D., Gomes, G. R. R., \& Costa, H. (2012). Mineração de texto e suas aplicações na literatura cientifica-Estudo bibliométrico. Exatas \& Engenharias, 2(04).

Zagato, L. (2019). Ainda é possível que os países em desenvolvimento façam seu catching up no século XXI? Brazilian Journal of Political Economy, 39(3), 527-543. 